\title{
MODELING THE PERFORMANCE OF BI-TEXTURED MICROPILLAR ARRAY AS A WICKED EVAPORATOR
}

\author{
Hassan Azarkish \\ Universite de Sherbrooke \\ Sherbrooke, Quebec, Canada
}

\author{
Amin Behzadmehr \\ University of Sistan and Baluchestan \\ Zahedan, Iran
}

\author{
Luc G. Frechette \\ Universite de Sherbrooke \\ Sherbrooke, Quebec, Canada
}

\begin{abstract}
In the present work, the performance of bi-textured micro pillar arrays has been modeled as a wicked evaporator to provide steam flow via the thin film evaporation mechanism. Bi-textured micro pillar evaporator consists of an array with rough hydrophilic pillar bases and smooth hydrophobic tips. Water wicks between the rough hydrophilic sections of the micro pillar array to cover the surface, and vaporizes from the thin films that are formed in the vicinity of the pillar walls. The stability of the phase change mechanism is increased due to the change in direction of the capillary forces at the roughsmooth interface of micro pillars. The experimental results show that the pure evaporation mechanism occurs for a surface temperature above saturation on the bi-textured micro pillar array. The numerical analysis shows that there are optimal micro pillar dimensions for each surface temperature. The evaporation mass flow rate at the optimum dimensions is higher than the pool boiling mass flow rate on a bare surface at the same surface temperature. However, the wicked evaporator performance decreases for larger evaporator sizes.
\end{abstract}

\section{INTRODUCTION}

Thin film evaporation and microchannel boiling are well known heat transfer mechanisms for high heat flux applications. They have been proposed and studied mainly for cooling of advanced semiconductor devices [1]. Another application for thin film evaporation is to provide a controllable flow of fully evaporated steam for micro chemical processing and micro heat engines [2, 3]. One approach consists of conducting the phase change in microchannels, but it has often lead to inconsistent vapor quality at the exit. Alternatively, shaped microchannels have been shown to provide fully evaporate steam, using surface tension and a streamwise temperature gradient [2,3]. Experimental results showed that a limited range of evaporation mass flow rate can be achieved with this method due to its limited liquid thin film area.

In addition to microchannels, much attention has been devoted in recent years on micro and nanostructured surfaces for phase change heat transfer enhancement [4]. Coso et al. [5] used biporous wick structures consisting of microscale pin fins separated by microchannels. Micropillars are used to generate high capillary suction as well as increasing the liquid thin film area, while microchannels are used to reduce overall flow resistance. Experimental data shows that heat fluxes up to 120 $\mathrm{W} / \mathrm{cm}^{2}$ can be dissipated when thin film evaporation is dominant. In the nucleate boiling dominated regime, heat fluxes of $277 \mathrm{~W} / \mathrm{cm}^{2}$ were dissipated by wicks with heaters of 1 $\mathrm{cm}^{2}$ area. However it is difficult to determine when the transition from evaporative to nucleate boiling occurs. An interesting point is that heat fluxes up to $733 \mathrm{~W} / \mathrm{cm}^{2}$ can be dissipated by wicks with smaller heater area $\left(0.0625 \mathrm{~cm}^{2}\right)$, showing the impact of size. It is also shown that the evaporation rate is significantly affected by the wick length in wick micro evaporators. Narayanan et al. [6, 7] have shown that high rate of evaporation can be achieved by using a nanoporous membrane, and that the thin film evaporation mechanism leads to high heat flux dissipation especially in small scales. 
Although these studies indicated that the micro and nanostructured surfaces lead to high phase change heat fluxes, they rarely address the challenges of providing high flow rates of steam without any pulsations and liquid droplets. Azarkish et al. [8] used micro structured, nanostructured and hybrid micro-nanostructured surfaces to evaluate these limitations on the different surfaces. Three main limitations are observed in experiments: (a) water spreading between micro and nanostructured surface, (b) forming the liquid droplet or films on the surface by merging thin films and (c) dynamic behavior of evaporation especially at high temperatures. Based on these limitations, we introduced a novel bi-textured micropillar array to increase the evaporation rate as well as access to more controllable system [9]. It consists of a rough hydrophilic section at the base of each pillar and smooth hydrophobic section at the top. Since the rough section of the micropillars is hydrophilic, water tends to wick well through the array and cover the surface. Also, changing the direction of capillary forces from the rough to the smooth sections of the pillars prevents upward movement of the water and consequent flooding of the surface. Water completely spreads between the rough sections of the microstructures and vaporizes without any water droplet formation on the surface. It shows that the smart and controllable surfaces for thin film evaporation can be achieved by changing the surface properties and the micro and nanostructure.

On the other hand, boiling is another high heat flux mechanism with some limitations especially in critical heat flux. The role of wickability on the critical heat flux of structured superhydrophilic surfaces was investigated by Rahman et al. [10]. They reported that the highest value of critical heat flux for water on the Structured Superhydrophilic Surfaces is about $257 \mathrm{~W} / \mathrm{cm}^{2}$. On another study, they used biconductive surfaces by embedding an array of low-conductive lines into a high-conductivity substrate to enhance the boiling heat transfer [11]. This creates an in-plane variation in surface temperature, which enhances the bubble dynamics and departure. Experimental results show that the boiling heat transfer coefficient has been increased five times at the same superheat temperature by using this bi-conductive surface. Also, it doubled the critical heat flux, from $116 \mathrm{~W} / \mathrm{cm}^{2}$ to 230 $\mathrm{W} / \mathrm{cm}^{2}$. These studies show the limitation of boiling heat transfer. The maximum critical heat flux is around 230-257 $\mathrm{W} / \mathrm{cm}^{2}$ which is close to the evaporation rate in the thin film evaporators shown to date [5-7]. Moreover, the performance of thin film evaporators can be enhanced by using bi-textured micro pillar arrays [9]. Therefore it seems that further increases in heat flux can be attained via thin-film evaporation on such surfaces.

In the present work, we study the limitations of the bitextured micropillar array for thin film evaporation. It will be shown that the full evaporation mechanism can be achieved for surface temperatures above saturation. The heat flux curve of boiling on the bare silicon surface is compared with the evaporation rate from typical bi-textured micropillar array to show its potential as an alternative heat transfer mechanism, especially at low level superheat (Surface temperature between $100^{\circ} \mathrm{C}<T_{s}<110^{\circ} \mathrm{C}$ ). Thin film evaporation modeling is applied to determine the maximum theoretical potential of evaporation on the bi-textured micropillar array for different pillar dimensions and different superheat levels. The viscus force of liquid flow between the micropillars is considered as the main limiting parameter. The maximum mass flow rate which can be wicked between micropillars is calculated based on the balance between capillary and viscous forces. This allows the prediction of a more realistic value of evaporation from micropillar arrays, compared to the ideal evaporation rate without the wicking limitation. The results will show that the thin film evaporation from bi-textured micro pillar arrays can be a good alternative to the boiling mechanism to increase the heat transfer rate as well as to have more controllable flows for small devices.

\section{BI-TEXTURED MICROPILLAR ARRAYS}

The SEM image of bi-textured micropillar array is shown in Fig. 1. It was fabricated on a silicon wafer based on two different DRIE recipes to form micropillars with rough bases and smooth tip sections, as described previously [9]. The microstructures are silicon micro columns of $4 \mu \mathrm{m} \times 4 \mu \mathrm{m}$ with a $50 \mu \mathrm{m}$ height $(20 \mu \mathrm{m}$ smooth and $30 \mu \mathrm{m}$ rough sections) and $4 \mu \mathrm{m}$ spacing were fabricated over $1 \mathrm{~cm}^{2}$ silicon surface.

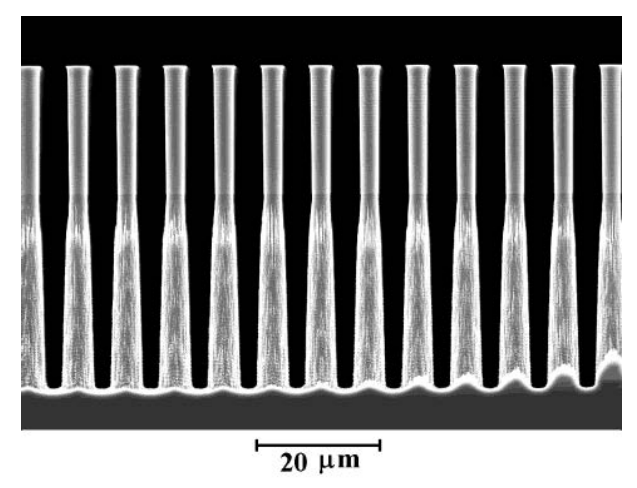

(a) Cross section view

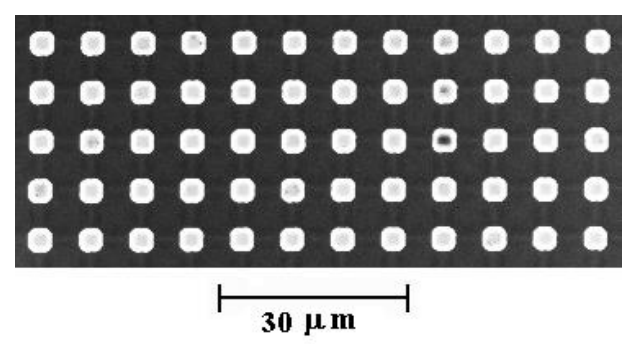

(b) Top view

Fig. 1. SEM images of a bi-textured micropillar array [9] 
Two mechanisms are considered to control and increase the evaporation rate. The first one consists of increasing the water spreading between micropillars by creating rough hydrophilic surfaces at the base of the micropillars. The second mechanism is to prevent the formation of water droplets or water films over the micropillars by changing the direction of capillary forces at the rough-smooth (hydrophilic-hydrophobic) interface. For this configuration, the dynamic behavior of evaporation (periodic rise and receding of the meniscus) occurs in the bottom part of the pillars (Fig 2a). Without the hydrophobic top portion of the pillars (Simple micropillar), the thin films can merge and form a single film across the entire surface (Fig $2 \mathrm{~b}$ ), greatly reducing its ability to evaporate.
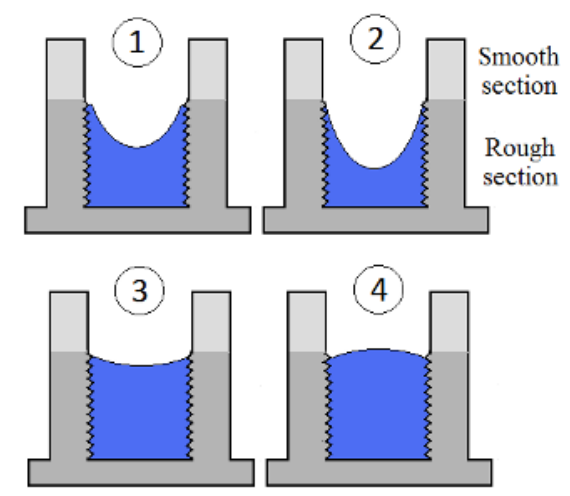

(a)

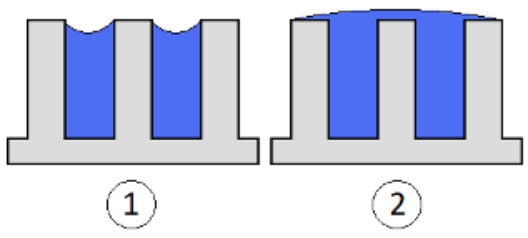

(b)

Fig. 2. Schematics of dynamic behaviour of evaporation:

(a) Bi-textured micropillar array

(b) Simple micropillar

\section{EXPERIMENTAL OBSERVATION}

To evaluate the capability of bi-textured micropillar arrays for steam production, thin film evaporation from these surfaces will be compared with boiling on a bare silicon surface for surface temperatures above saturation. The experimental results are based on the author's previous work on the evaporation from bi-textured micropillar arrays [9] and boiling on the micro and nanostructured surfaces [8]. Evaporation or boiling rates are equal to the entrance water mass flow rate in steady state, which is measured and controlled by a syringe pump (Harvard Apparatus PHD $22 / 2000$ ) with $\pm 1 \%$ accuracy. The chip is heated with a cartridge heater in a custom packaging, and surface temperature is measured by a thermocouple (Type K) within $\pm 1{ }^{\circ} \mathrm{C}$. The surfaces are initially maintained at the given temperature; the small increment on the mass flow rate is applied until stabilization. More details on the experimental test set-up and procedure are presented elsewhere [8, 9].

Figure 3 compares the boiling curve of water on a bare silicon surface with the evaporation heat flux curve on the bitextured micropillar array. An interesting point is the thin film evaporation heat flux on the bi-textured pillar surface is higher than boiling heat flux on a bare silicon surface for a surface temperature $T s<109^{\circ} \mathrm{C}$. Therefore thin film evaporation mechanism on bi-textured surface is more efficient than the pool boiling mechanism on the bare silicon surface. As shown, a deflection is observed on the boiling curve of bare silicon at $T s \approx 105^{\circ} \mathrm{C}$, which corresponds to the onset of nucleate boiling (ONB). Free convection boiling exists for $T_{s}<105^{\circ} \mathrm{C}$ and nucleate boiling is exist for $T s>105^{\circ} \mathrm{C}$. However there is no deflection on the evaporation curve on the bi-textured surface even for the surface temperatures below the saturated temperature. It indicates that there is a unique phase change mechanism (thin film evaporation) in the temperature range of $99^{\circ} \mathrm{C}<T_{s}<111^{\circ} \mathrm{C}$.

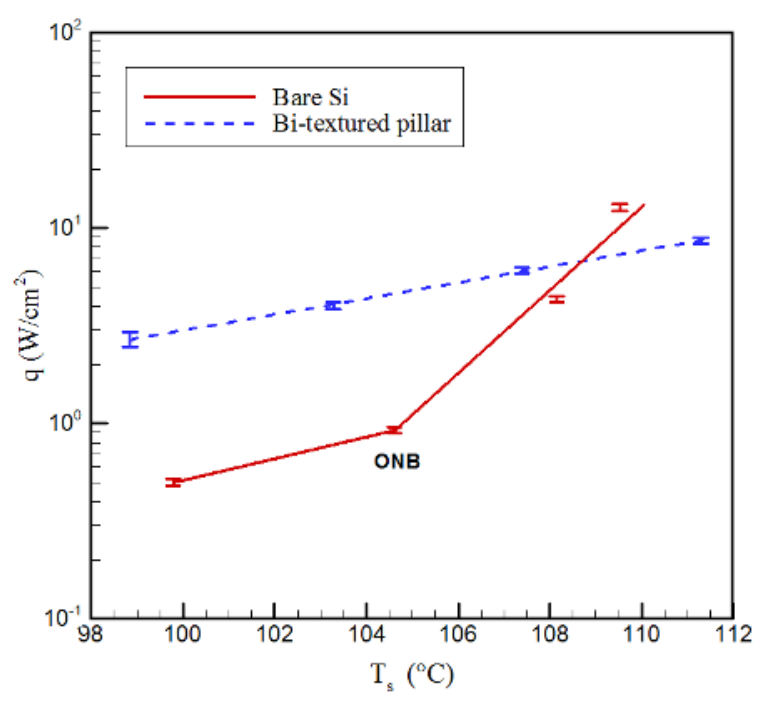

Fig. 3. Comparison of the water boiling curve on a bare silicon surface with the evaporation heat flux curve on the bi-textured micropillar array.

Since these results were for a single geometry, it is expected that the performance of the bi-textured micropillar array may be enhanced by finding the optimum dimension of microstructures. In the present work, a numerical modeling will be conducted to determine the optimum dimension of bitextured micropillar array. 


\section{MODELING}

A thin film evaporation model is used to calculate the maximum potential of evaporation from the micropillar array. It is considered that liquid spreads uniformly throughout the micropillars and thin liquid films are formed at the roughsmooth interface. The augmented Young-Laplace equation represents the pressure difference between the liquid and the vapor zones $\left(P_{v^{-}} P_{l}\right)$ at the liquid-vapor interface as the sum of the capillary and disjoining pressures $[12,13]$ :

$$
P_{v}-P_{l}=P_{c}+P_{d}
$$

The capillary pressure is caused by the curvature of the meniscus and can be expressed as:

$$
P_{c}=\sigma \frac{d^{2} \delta}{d x^{2}}\left[1+\left(\frac{d \delta}{d x}\right)^{2}\right]^{-3 / 2}
$$

where $\sigma$ and $\delta$ are the liquid-vapor interfacial surface tension and the film thickness respectively. The disjoining pressure, $P_{d}$, is caused by intermolecular and surface forces through the thin film. For non-polar liquids, the disjoining pressure can be calculated by considering the effect of Van der Waals forces as the main intermolecular force. However, the effects of other intermolecular forces on the disjoining pressure cannot be negligible for polar liquids such as water. Azarkish et al. [14] proposed a modified disjoining pressure model to take into account the effect of other intermolecular forces by multiplying a non-dimensional stretching function to the van der Waals disjoining pressure:

$$
P_{d}=P_{d, v d W}\left(\frac{\xi}{\delta}\right)^{n}=\frac{A}{\delta^{3}}\left(\frac{\xi}{\delta}\right)^{n}
$$

where $A=2.87 \times 10^{-21} \mathrm{~J}$. In the present work, the constants $\xi$ and $n$ are considered $\xi=1 m$ and $n=0.1$ for water on the silicon surface. By assuming uniform vapor pressure along the meniscus, combining equations 1 and 2 and differentiating with respect to $x$ :

$$
\delta^{\prime \prime \prime}-\frac{3 \delta^{\prime} \delta^{\prime 2}}{1+\delta^{\prime 2}}+\frac{1}{\sigma}\left(\frac{d P_{l}}{d x}-\frac{d P_{d}}{d x}\right)\left(1+\delta^{\prime 2}\right)^{3 / 2}=0
$$

The liquid pressure gradient can be calculated by lubrication theory as a function of the local evaporative mass flux:

$$
\frac{d P_{l}}{d x}=\frac{3 v}{\delta^{3}} \int_{-\infty}^{x} m^{\prime \prime} d x
$$

Wayner et al. [15] presented a relation to calculate the evaporative mass flux for small superheat and small changes in vapor pressure as:

$$
m^{\prime \prime}=a\left(T_{l v}-T_{v}\right)-b\left(P_{d}+P_{c}\right)
$$

where

$$
a=C\left(\frac{M}{2 \pi \bar{R} T_{l v}}\right)^{1 / 2} \frac{P_{v} M h_{f g}}{\bar{R} T_{v} T_{l v}} \quad b=C\left(\frac{M}{2 \pi \bar{R} T_{l v}}\right)^{1 / 2} \frac{P_{v} V_{l}}{\bar{R} T_{l v}}
$$

However, $T_{l v}$ is unknown. Under steady-state conditions, the local conduction heat flux through the liquid film is equal to the evaporation heat flux [12]:

$$
m^{\prime \prime} h_{f g}=\frac{k_{l}\left(T_{w}-T_{l v}\right)}{\delta}
$$

so by combining equations 6 and $7, T_{l v}$ and $m^{\prime \prime}$ can be calculated as [16]:

$$
\begin{gathered}
T_{l v}=\frac{1}{a+\frac{k_{l}}{\delta h_{f g}}}\left\lfloor k_{l} \frac{T_{w}}{\delta h_{f g}}+a T_{v}+b\left(P_{d}+P_{c}\right)\right\rfloor \\
m^{\prime \prime}=\frac{1}{1+\frac{a \delta h_{f g}}{k_{l}}}\left[a\left(T_{w}-T_{v}\right)-b\left(P_{d}+P_{c}\right)\right]
\end{gathered}
$$

The fourth order Runge-Kutta method is used to solve the initial-value problem of Eq. (4) with boundary conditions:

$$
\begin{gathered}
\left.\delta\right|_{x=0}=\delta_{0} \\
\left.\delta^{\prime}\right|_{x=0}=\text { finite } \\
\left.\delta^{\prime \prime}\right|_{x=0}=0
\end{gathered}
$$

More detailed information about these boundary conditions and solving method of the Eq. (4) are presented elsewhere $[3,13,17]$.

Permeability is a key parameter to determine and optimize the liquid flow rate through the micropillar array. Hale et al. [18] had a comprehensive study on the micropillar array permeability by comparing several permeability models including Yazdchi et al. [19], Tamayol and Bahrami [20], Xiao et al [21] and an analytical solution derived from a 2dimensional velocity profile with appropriately varying boundary conditions. In the present work, a combination of Xiao with Yazdchi's models is used to predict the micropillar array permeability [18]:

$$
K_{B}^{*}=K_{Y}^{*}-\frac{\left(K_{Y}^{*}\right)^{3 / 2}}{\phi_{1} \sqrt{1-\frac{\pi}{4} \phi_{2}^{-2}}} \tanh \left(\frac{\phi_{1}}{\sqrt{K_{Y}^{*}}} \sqrt{1-\frac{\pi}{4} \phi_{2}^{-2}}\right)
$$

where

$$
\begin{gathered}
K_{Y}^{*}=\frac{K_{G}^{*}}{1+0.336\left(\frac{\pi}{4}-\phi\right)}+\left(K_{D}^{*}-\frac{K_{G}^{*}}{1+0.336\left(\frac{\pi}{4}-\phi\right)}\right) \frac{1+\tanh \left(\frac{0.25-\phi}{0.037}\right)}{2} \\
K_{G}^{*}=\frac{4}{9 \pi \sqrt{2}}\left(\phi_{2}-1\right)^{5 / 2} \\
K_{D}^{*}=\frac{1}{32 \phi}\left(\ln \left(\frac{1}{\phi}\right)-1.476+\frac{2 \phi-0.796 \phi^{2}}{1+0.489 \phi-1.60 \phi^{2}}\right) \\
\phi=\frac{\pi}{4} \phi_{2}^{-2} \quad \phi_{2}=l / d
\end{gathered}
$$

The mass flow rate between micropillars can be calculated by balancing the capillary and viscus forces as:

$$
\dot{m}=\frac{\rho A d^{2}}{\mu L} \Delta P_{c a p} K_{B}^{*}
$$


where $L$ is wick length, $A$ is cross-sectional wick area and $\Delta P_{\text {cap }}$ is capillary force:

$$
\Delta P_{\text {cap }}=\frac{\pi d \sigma \cos (\theta)}{l^{2}-\pi d^{2} / 4}
$$

The ideal evaporation rate on the bi-textured micropillar array can be calculated by solving Eq. (4) with boundary conditions of Eqs. (10a-c). The wicked liquid flow rate between micropillars is obtained by solving the Eq. (17) based on the micropillar dimensions and wicking length. The comparison between these values leads to a more realistic prediction of the evaporation rate for different dimensions and superheat level.

\section{MODELING RESULTS AND DISCUSSION}

A square layout of the micropillar array with pillar diameter $d$ and pillar pitch of $2 d$ is considered as a case study. The maximum ideal evaporation rate which can occur by thin film evaporation from the micropillar array (based on Eq. 4) is presented in Fig. 4. As seen, the evaporation rate has been strongly dependent on the pillar dimensions for pillar diameters less than $10 \mu \mathrm{m}$. The ideal evaporation rate is more than $1000 \mathrm{~W} / \mathrm{cm}^{2}$ at any superheat level for pillar diameters less than $1 \mu \mathrm{m}$; it decreases rapidly with increasing pillar diameter, to reach $100-500 \mathrm{~W} / \mathrm{cm}^{2}$ for a pillar diameter of 10 $\mu \mathrm{m}$ at different superheat levels between $1-10^{\circ} \mathrm{C}$. The variation of evaporation rate is not significant for pillar diameters more than $20 \mu \mathrm{m}$. At this condition, the effect of superheat level dominates on the evaporation rate.

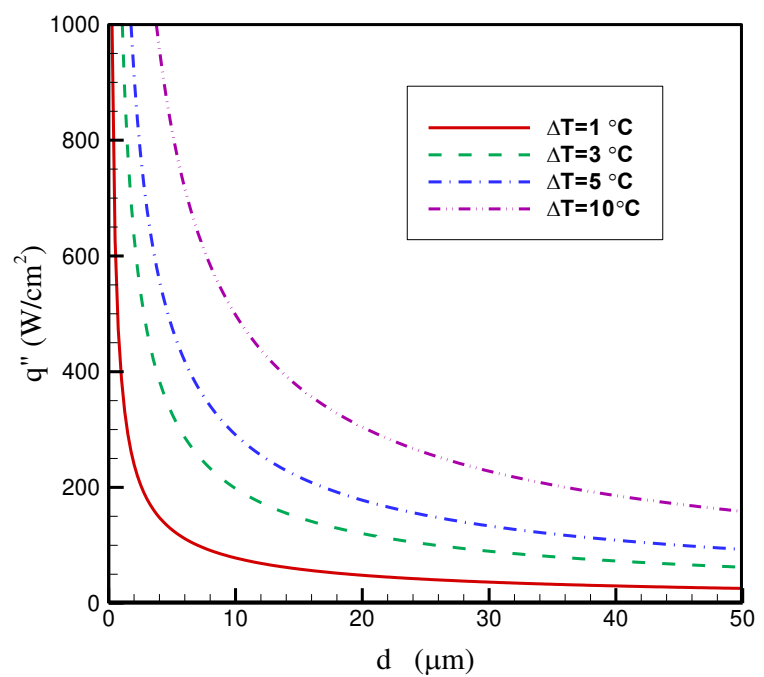

Fig.4. Ideal rate of evaporation as a function of pillar diameter and superheat temperature.

The real evaporation rate will however be limited by the balance between viscous forces and capillary pressure. In this case, the wick flow rate can be calculated by solving Eq. (17). The equivalent evaporation heat flux that corresponds to the wick flow is presented in Fig. 5. As shown, significant heat flux can be achieved for wick length of less than $2 \mathrm{~mm}$. However by increasing the overall dimension of micro evaporator, the wick limitation becomes dominant. For example, the evaporation rate is less than $50 \mathrm{~W} / \mathrm{cm}^{2}$ for wick length more than $4 \mathrm{~mm}$. It suggests that the wick flow is a key limiting parameter for micro evaporator with wick length of more than $4 \mathrm{~mm}$.

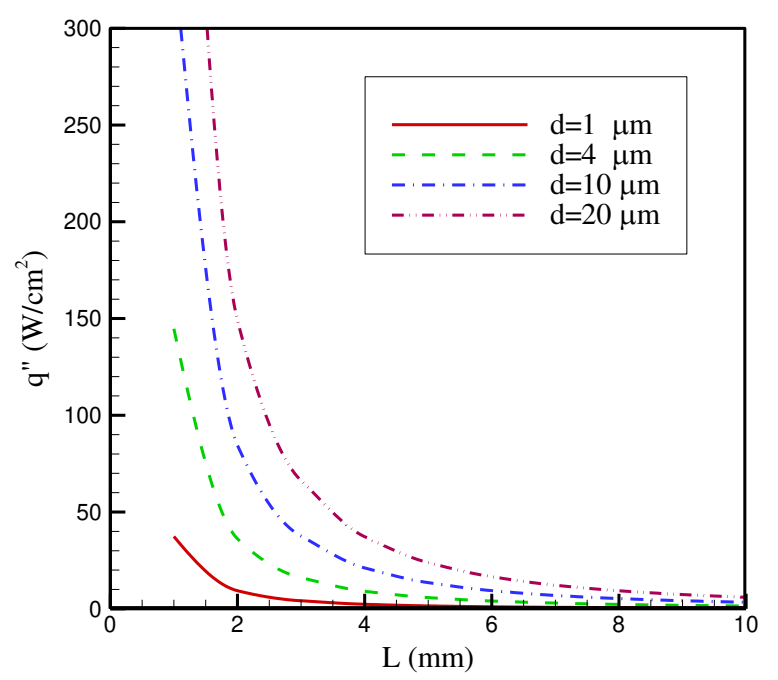

Fig. 5. Equivalent evaporation heat flux due to the wicking phenomena as a function of wick length and pillar diameter.

The height of pillar's rough section is $30 \mu \mathrm{m}$.

Unlike the ideal evaporation rate, wick flow rate decreases by decreasing the pillar diameter. This trade-off therefore leads to an optimum micropillar dimension for each superheat level. The comparison between ideal evaporation rate and wick flow rate is presented on Fig. 6 for different superheat levels. The intersection of two curves represents the optimum conditions for each superheat temperature and wick length. As shown, the optimum diameter of pillars is a function of superheat temperature and overall dimension of the micro evaporator (wick length). For a given superheat temperature, the maximum heat flux decreases and the optimum pillar diameter increases with increasing wick length. Very high heat flux is predicted for some specific conditions. It is more than 100 $\mathrm{W} / \mathrm{cm}^{2}$ for a wicking length of less than $2 \mathrm{~mm}$, which is much higher than pool boiling heat flux on the bare silicon surface. As shown in fig. 3, the measured experimental heat flux value for pool boiling of water on the bare silicon surface is about $20 \mathrm{~W} / \mathrm{cm}^{2}$ for $10{ }^{\circ} \mathrm{C}$ superheat. However, the evaporation rate 
decreases rapidly with increasing wick length, so for a wick length of $7 \mathrm{~mm}$, the evaporation rate is less than $20 \mathrm{~W} / \mathrm{cm}^{2}$.

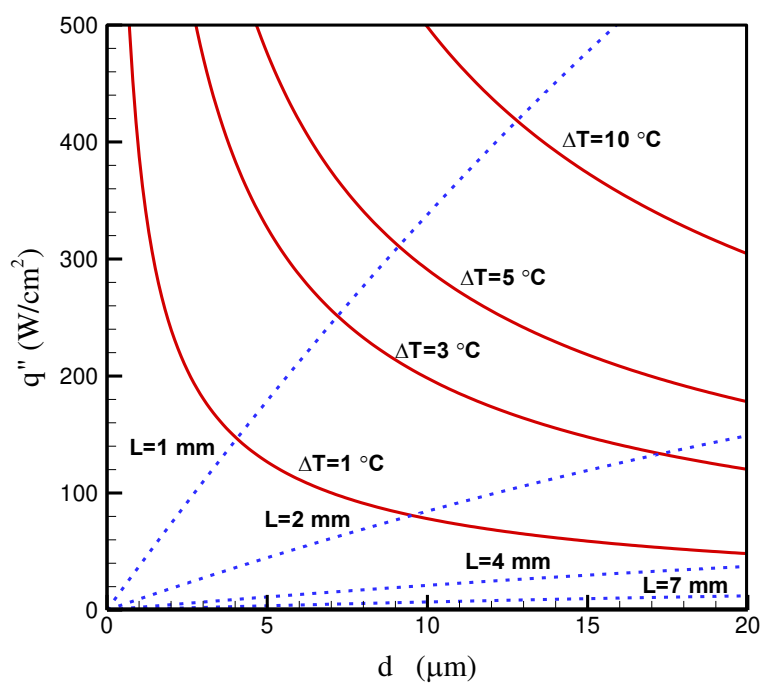

Fig. 6 . Comparison between ideal evaporation rate (solid lines) and wick flow rate (dash line). The height of the pillar's rough section is $30 \mu \mathrm{m}$.

The depth of the rough section of the micropillar array is another parameter affecting the evaporation rate from bitextured micropillar arrays. The wicking limit is changed by changing the depth of pillars. Figure 7 shows the comparison between ideal evaporation rate and wick flow rate when the height of pillar's rough section is $50 \mu \mathrm{m}$. Compared to $30 \mu \mathrm{m}$ height (Fig. 6), it is obvious that the evaporation rate increases with increasing the depth of pillars due to facilitated wicking. However from a fabrication point of view, the appropriate aspect ratio of pillar depth to the pillar spacing should be less than 10. Then the depth of micropillars is limited by fabrication process flow, especially for thin pillars.

An interesting point is that the wick length is a limiting parameter on the evaporation rate of bi-textured micropillar arrays. However the nucleation sites, bubble dynamic and flow pattern are the dominant parameters in pool boiling. In an experimental study, Rahman et al. [11] reach the boiling heat flux of $150 \mathrm{~W} / \mathrm{cm}^{2}$ on a modified cupper surface for $10{ }^{\circ} \mathrm{C}$ superheat. It is five times more than the pool boiling heat flux on the bare cupper surface at the same superheat temperature. They modified the flow pattern of vapor bubbles, a limiting parameter. The departure diameter of vapor bubbles during pool boiling was reported at about $2.33 \mathrm{~mm}$. It corresponds to the wick length of $1.17 \mathrm{~mm}$, by considering the wick length is equal to the departure radius. It is suggested that the rate of pool boiling heat transfer can be enhanced to $400 \mathrm{~W} / \mathrm{cm}^{2}$ by using bi-textured micro pillar array (intersection of the lines for $\mathrm{L}=1 \mathrm{~mm}$ and $\Delta T=10^{\circ} \mathrm{C}$ in Fig. 6). However more experimental and numerical efforts will be needed in the future to prove this suggestion.

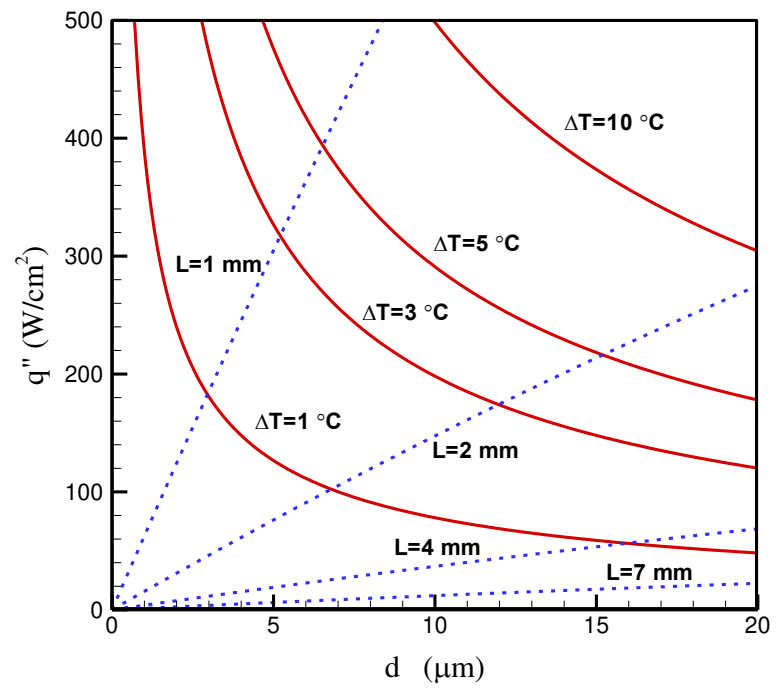

Fig. 7 . Comparison between ideal evaporation rate (solid lines) and wick flow rate (dash line). The height of the pillar's rough section is $50 \mu \mathrm{m}$.

\section{CONCLUSION}

In the present study, the performance of bi-textured micro pillar arrays has been investigated to provide a vapor flow using the thin film evaporation mechanism instead of more commonly used boiling. Experimental results for typical bitextured micro pillar arrays $(4 \mu \mathrm{m} \times 4 \mu \mathrm{m}$ micro columns with $20 \mu \mathrm{m}$ smooth and $30 \mu \mathrm{m}$ rough sections) show that the phase change mechanism is thin film evaporation and it does not change for surface temperatures from $99^{\circ} \mathrm{C}<T_{s}<111^{\circ} \mathrm{C}$. Also the evaporation rate by thin film evaporation on the bi-textured micropillar arrays for this typical dimension is predicted to be more than the boiling heat flux on a bare silicon surface with $T s<109^{\circ} \mathrm{C}$.

The performance of bi-textured micropillar arrays was modeled to determine optimal configurations. The ideal evaporation rate was calculated based on thin film evaporation modeling and compared with the maximum flow rate achieved by wicking. Results show that the wick length is a key parameter on the performance of micropillar array. High heat fluxes are predicted for wick lengths of less than $2 \mathrm{~mm}$. Also the evaporation rate from thin film evaporation is expected to be more than the boiling heat flux on a bare surface for superheat less than $10^{\circ} \mathrm{C}$ and wick lengths less than $7 \mathrm{~mm}$. 


\section{NOMENCLATURE}

A

$d$

$h_{f g}$

$K^{*}$

$k$

$\mathrm{L}$

M

$m^{\prime \prime}$

$P$

$\bar{R}$

$T$

V

$x$

Greek symbols

$\delta$

$\delta^{\prime}, \delta^{\prime \prime}, \delta^{\prime \prime \prime}$

$\mu$

$v$

$\rho$

$\sigma$

Subscripts

$c$

d

l

lv

\section{sat}

$v$

w

\section{REFERENCES}

[1] Plawsky J. L., Fedorov A. G., Garimella S. V., Ma H.B., Maroo S.C., Chen L., Nam Y., 2014, Nano and microstructures for thin-film evaporation-a review Nanoscale Microscale Thermophys. Eng., Vol. 18, pp. 251-69.

[2] Arslan S., Frechette L.G., 2009, Controlled flow evaporation in complex microchannels with nonuniform wall temperatures, in: Proc. ASME Int. Mech. Eng. Congr. Expo. Florida.

[3] Azarkish H., Arslan S., Behzadmehr A., Sheikholeslami T. F., Sarvari S.M.H., Fréchette L.G., 2015, Experimental and numerical investigation of a shaped microchannel evaporator for a micro Rankine cycle application, Int. J. Therm. Sci., Vol. 96, pp. 191-200.

[4] McCarthy M., Gerasopoulos K., Maroo S.C., Hart A.J., 2014, Materials, Fabrication, and Manufacturing of Micro/Nanostructured Surfaces for Phase-Change Heat Transfer Enhancement, Nanoscale Microscale Thermophys. Eng., Vol. 18, pp. 288-310.
[5] Coso D., Srinivasan V., Lu M.C., Chang J.Y., Majumdar A., 2012, Enhanced Heat Transfer in Biporous Wicks in the Thin Liquid Film Evaporation and Boiling Regimes, ASME, J. Heat Transfer, Vol. 143, pp. 101501,1-11.

[6] Narayanan S., Fedorov A.G., Joshi Y.K., 2013, Heat and mass transfer during evaporation of thin liquid films confined by nanoporous membranes subjected to air jet impingement, Int. J. Heat Mass Transfer, 58 (2013) 300-311.

[7] Narayanan S., Fedorov A.G., Joshi Y.K., 2011, Interfacial Transport of Evaporating Water Confined in Nanopores, Langmuir, Vol. 27, pp. 10666-10676.

[8] Azarkish H., Behzadmehr A., Sheikholeslami T. F., Sarvari S.M.H., Fréchette L.G., 2015, Water evaporation phenomena on micro and nanostructured surfaces, Int. J. Therm. Sci., Vol. 90, pp. 112-121.

[9] Azarkish H., Behzadmehr A., Sheikholeslami T. F., Sarvari S.M.H., Fréchette L.G., 2014, A novel silicon bitextured micropillar array to provide fully evaporated steam for a micro-Rankine cycle application, J. Phys. D: Appl. Phys., Vol. 47, pp. 475301.

[10] Rahman M., Olceroglu E., Mccarthy M., 2014, Role of Wickability on the Critical Heat Flux of Structured Superhydrophilic Surfaces, Vol. 30, pp. 11225-11234.

[11] Rahman M., Pollack J., Mccarthy M., 2015, Increasing Boiling Heat Transfer using Low Conductivity Materials, Scientific reports, vol. 5, pp. 13145.

[12] Faghri A., Zhang Y., 2006, Transport phenomena in multiphase systems, Elsevier, USA.

[13] Wang H., Garimella S.V., Murthy J.Y., 2007, Characteristics of an evaporating thin film in a microchannel, Int. J. Heat Mass Transfer, Vol 50, pp. 3933-3942.

[14] Azarkish H., Behzadmehr A., Frechette L.G., Sheikholeslami T.F., Sarvari S.M.H., 2013, A modified disjoining pressure model for thin film evaporation of water, Proc. ASME Int. Mech. Eng. Congr. Expo. San Diago.

[15] Wayner Jr. P.C., Kao Y.K., LaCroix L.V., 1976, The interline heat transfer coefficient of an evaporating wetting film, Int. J. Heat Mass Transfer, Vol.19, pp. 487-492.

[16] Do K.H., Kim S.J., Garimella S.V., 2008, A mathematical model for analyzing the thermal characteristics of a flat micro heat pipe with a grooved wick, Int. J.Heat Mass Transfer, Vol. 51, pp. 4637-4650.

[17] Zhao J.J., Duan Y.Y., Wang X.D., Wang B.X., 2008, Effects of superheat and temperature-dependent thermophysical properties on evaporating thin liquid films in microchannels, Int. J.Heat Mass Transfer, Vol. 54, pp. 12591267.

[18] Hale R.S., Bonnecaze R.T., Hidrovo C.H., 2014, Optimization of capillary flow through square micropillar arrays, Int. J. Multiphase Flow, Vol. 58, pp. 39-51.

[19] Yazdchi K., Srivastava S., Luding S., 2011, Microstructural effects on the permeability of periodic fibrous porous media, Int. J. Multiphase Flow, Vol. 37, pp. 956-966. 
[20] Tamayol A., Bahrami M., 2009, Analytical determination of viscous permeability of fibrous porous media. Int. J. Heat Mass Transfer, Vol. 52, pp.2407-2414.
[21] Xiao R., Enright R., Wang E.N., 2010, Prediction and optimization of liquid propagation in micropillar arrays, Langmuir, Vol. 26, pp. 15070-1 